\title{
Can Black Holes Decay to Naked Singularities?
}

\author{
Saurya Das ${ }^{\sharp}$, J. Gegenberg ${ }^{\dagger}$, and V. Husain ${ }^{\dagger}$ \\ \# Dept. of Physics, University of Winnipeg \\ Winnipeg, Manitoba, Canada R3B 2E9 \\ $\dagger^{\dagger}$ Dept. of Mathematics and Statistics, University of New Brunswick \\ Fredericton, New Brunswick, Canada E3B 5A3
}

\begin{abstract}
We investigate thermodynamic properties of two types of asymptotically antide Sitter spacetimes: black holes and singular scalar field spacetimes. We describe the possibility that thermodynamic phase transitions can transform one spacetime into another, suggesting that black holes can radiate to naked singularities.
\end{abstract}

*This essay received an "honorable mention" in the 2001 Essay Competition of the Gravity Research Foundation - Ed.

†emails: saurya@theory.uwinnipeg.ca, lenin@math.unb.ca, husain@math.unb.ca 
The discovery of the connection between semi-classical properties of black holes and thermodynamics has naturally led to the application of statistical physics ideas to black hole physics. The main questions concern the origin of black hole microstates and how these might arise from a quantum gravity theory, the final state of Hawking radiation, and the possibility of thermal phase transitions between black holes and other spacetimes.

In this essay we discuss the last of these issues in the context of asymptotically anti-deSitter (AAdS) spacetimes. This class of spacetimes is of current interest in the context of the $\mathrm{AdS} / \mathrm{CFT}$ conjecture in string theory.

A class of phase transitions for these spacetimes was studied by Hawking and Page [回]. They showed that the canonical ensemble exists, and that at a given temperature above a certain minimum, there are three possible states: a low mass black hole, a high mass black hole and thermal radiation in AdS space. The first of these states has negative specific heat and can decay to the other configurations, which are stable but not equally probable.

These thermodynamic results can be extended to arbitrary spacetime dimensions, and related to the confinement/deconfinement phase transition in a dual conformal Yang-Mills theory [2]. Similar phase transitions also occur between constant curvature black holes, whose horizon topologies are other than spherical, and the AdS soliton spacetime [3].

In all of these cases the possibility of a phase transition is indicated by a simple calculation: the Euclidean action difference $\Delta I$ of two spacetimes, which gives the probability ratio of the two configurations. If $\Delta I$ changes 
sign as the metric parameters are varied, it is an indication that one phase becomes more probable than the other at a fixed temperature (determined by appropriate matching of Euclidean time periodicities).

We consider the Einstein-scalar field system $\left(g_{a b}, \phi\right)$, with minimal scalar field coupling, and show that there is a phase transition between black holes and naked singularities using arguments analogous to those used in Ref. [1]. We focus on three dimensional spacetimes for which the details are particularly simple.

The two Euclidean spacetimes of interest for this study are the BTZ black hole

$$
d s^{2}=\left((r / l)^{2}-M\right) d t^{2}+\left((r / l)^{2}-M\right)^{-1} d r^{2}+r^{2} d \theta^{2}
$$

and the scalar field spacetime [4]

$$
\begin{aligned}
d s^{2} & =(x-b / 2)^{(1+a) / 2}(x+b / 2)^{(1-a) / 2} d t^{2}+\frac{l^{2}}{4\left(x^{2}-b^{2} / 4\right)} d x^{2} \\
& +\ell^{2}(x-b / 2)^{(1-a) / 2}(x+b / 2)^{(1+a) / 2} d \theta^{2}
\end{aligned}
$$

with

$$
\phi(x)=\frac{1}{2} \sqrt{\frac{1-a^{2}}{2}} \ln \left(\frac{x-b / 2}{x+b / 2},\right)
$$

where $\ell$ is cosmological constant length scale. The latter solution with $a=1$ is a BTZ black hole with $M_{B H}=b$. This makes the two metrics particularly easy to compare.

To calculate the action difference $\Delta I$ for these two solutions one integrates out to a fixed value of the 'radial' coordinate $x=X$, ensuring that the Euclidean time periodicities for the $t$ integrals are fixed so that the local 
temperatures are equal there: $\beta_{S C} \sqrt{-g_{00}^{S C}}=\beta_{B H} \sqrt{-g_{00}^{B H}}$. The limit $X \rightarrow \infty$ is taken at the end. The scalar matter terms must of course be included in the $\Delta I$ calculation.

At fixed temperature the action difference is

$$
\Delta I:=I_{S C}-I_{B H}=\frac{\pi^{2} l^{2}}{2 r_{+}}\left(\left(r_{+} / l\right)^{2}-\sqrt{2} k\right),
$$

where $r_{+}:=\sqrt{M} l$ is the BTZ black hole radius, and $\sqrt{2} k=b \sqrt{1-a^{2}}$ is the scalar field strength (3). This is finite even though there is a naked singularity at the origin for the scalar field solution! $\Delta I$ changes sign depending on the size of the black hole $r_{+}$in comparison with the scalar field strength $k$. This indicates a phase transition: sufficiently small black holes phase transform semi-classically to naked singularities!

This calculation suggests an outline (at the semi-classical level) of the entire process of gravitational collapse and its subsequent evolution. A scalar field collapsing in an AdS spacetime will form a black hole if appropriate initial conditions are satisfied [5]. The black hole will then radiate away its mass via Hawking radiation. When the mass is sufficiently small, of the order $k$, a phase change will occur to the scalar field configuration characterized by $k$. This is a static geometry with a naked singularity.

Several interesting questions can be asked at this stage. If the (AdS) $\leftrightarrow$ (Schwarzschild-AdS) transition reflects the (confinement) $\leftrightarrow$ (deconfinement) phase change in the boundary Yang-Mills theory, what is the correspondence for the (black hole) $\leftrightarrow$ (naked singularity) transition described above? Are there certain exotic states in the boundary conformal field theory which are 
dual to naked singularities in the bulk? Is a naked singularity indeed the final state of gravitational collapse in this model as suggested by our semiclassical argument? While some of these issues can be investigated in the currently available framework, the resolution of the others, such as those related to the end state of collapse would require one to go beyond the semi-classical regime to a full quantum theory of gravity.

\section{References}

[1] S.W. Hawking and D.N. Page, Comm. Math. Phys. 87, 577 (1983).

[2] E. Witten, Adv. Theor. Math. Phys. 2, 505 (1998) (hep-th/9803131).

[3] S. Surya, K. Schleich and D.M. Witt, 'Phase Transitions for Flat adS Black Holes', hep-th/0101134.

[4] S. Das, J. Gegenberg and V. Husain, 'Scalar Field spacetimes and AdS/CFT conjecture', hep-th/0101169.

[5] F. Pretorius and M.W. Choptuik, Phys. Rev. D 62 (2000) 124012 (grqc/0007008).

[6] V. Husain and M. Olivier, Class. Quant. Grav. 18 (2001) L1 (gr$\mathrm{qc} / 0008060)$. 